\title{
Centralized coordinated scheduling in LTE-Advanced networks
}

\author{
Oscar D. Ramos-Cantor ${ }^{*}$, Jakob Belschner ${ }^{2}$, Ganapati Hegde ${ }^{1}$ and Marius Pesavento ${ }^{1}$
}

\begin{abstract}
This work addresses the problem associated with coordinating scheduling decisions among multiple base stations in an LTE-Advanced downlink network in order to manage inter-cell interference with a centralized controller. To solve the coordinated scheduling problem, an integer non-linear program is formulated that, unlike most existing approaches, does not rely on exact channel state information but only makes use of the specific measurement reports defined in the 3GPP standard. An equivalent integer linear reformulation of the coordinated scheduling problem is proposed, which can be efficiently solved by commercial solvers. Extensive simulations of medium to large-size networks are carried out to analyze the performance of the proposed coordinated scheduling approaches, confirming available analytical results reporting fundamental limitations in the cooperation due to out-of-cluster interference. Nevertheless, the schemes proposed in this paper show important gains in average user throughput of the cell-edge users, especially in the case of heterogeneous networks.
\end{abstract}

Keywords: 4G mobile communication, Scheduling algorithms, Integer linear programming

\section{Introduction}

Interference is one of the main limiting factors of today's cellular communication networks in terms of user and network throughputs, especially when operating with full frequency reuse to achieve high spectral efficiency [1-4]. In modern cellular networks, the demand for high data rates is constantly increasing [5], with the users expecting to enjoy excellent network performance irrespective of their geographic location and the load conditions of the network. Thus, new solutions are required in order to fulfill the ever increasing requirements, in particular for the users located at the cell-edge suffering from large path loss and strong inter-cell interference. Promising advances in this aspect have been made with multi-antenna technology [6-9], network densification with interference management schemes [10-12], and Coordinated Multi-Point (CoMP) transceiver techniques [13, 14].

In this work, CoMP network operation is studied, where the base stations (BSs) are prompted to cooperate with each other with the objective of improving the overall

*Correspondence: oramos@nt.tu-darmstadt.de

${ }^{1}$ Communication Systems Group, Darmstadt University of Technology, 64283 Darmstadt, Germany

Full list of author information is available at the end of the article network performance, even at the expense of their individual cell or user throughputs [15]. In the literature, three main CoMP schemes are considered for the downlink scenario $[16,17]$. These are (i) Joint Transmission (JT), where multiple BSs simultaneously transmit a common message to a user equipment (UE), usually located at the cell-edge, (ii) Dynamic Point Selection (DPS), where at each transmission time, the UE can be served by a different BS without triggering handover procedures, and (iii) Coordinated Scheduling (CS), where the BSs jointly make the scheduling decisions in order to manage the interference experienced by the UEs in the cooperation cluster [18]. This paper focuses on the last CoMP scheme.

The performance of the abovementioned CoMP schemes heavily depends on the channel state information (CSI) available at the transmitter. This CSI can be of different types such as instantaneous channel coefficients or user's average achievable downlink data rates, among others. In practical downlink networks, where perfect global knowledge of the instantaneous channel coefficients is not available at the BSs, CSI is typically obtained in form of achievable data rate measurement reports generated by the UEs, averaged over multiple time/frequency/space dimensions and quantized to reduce the signaling overhead. In this work, the CoMP problem formulation is 
based on practical considerations of the CSI, in form of periodic achievable data rate measurement reports, in the following referred to as CSI reports.

The network architecture, in which the CoMP schemes are implemented, also influences the performance of such schemes. Two main CoMP network architectures are typically considered, namely, centralized and decentralized [19]. In the case of centralized CoMP, a central controller is connected to multiple BSs via backhaul links. This central controller is in charge of gathering and using the CSI reports, in order to make a coordinated decision among the connected BSs. For the decentralized CoMP case, decisions are individually made by each BS based on the information exchanged with neighboring BSs. In the case of centralized CoMP, high coordination gains are achievable at the expense of high computational complexity and large signaling overhead. On the other hand, decentralized CoMP requires significantly less information exchange with lower coordination gains. This work focuses on centralized CoMP.

Over the past years, important research has been carried out regarding CoMP schemes under different network architectures and CSI assumptions. In [20] and [21], JT and DPS schemes based on the enhanced CSI reports supported by Long Term Evolution (LTE)-Advanced Release 11, in the following denoted as $\mathrm{CSI}_{\text {lte }}^{\mathrm{R}-11}$ reports, have been investigated. The results therein show throughput gains for the cell-edge users mainly, and the possibility to improve mobility management by means of DPS. Barbieri et al. studied CS as a complement of enhanced inter-cell interference cancellation (eICIC) in heterogeneous networks in [22]. In their scheme, cooperation takes place in form of CS supported by beamforming in order to mitigate the interference caused by the macro BSs, to the UEs connected to the small cells. Multiple CSI reports are generated, where all possible precoders the macro BS can select from a finite precoder codebook are considered for the cooperation. The results present negligible gain for eICIC with CS, in comparison to eICIC-only. In [23], a cloud-radio access network (C-RAN) architecture is used for centralized CoMP JT in heterogeneous networks, which enables the cooperation of larger cluster sizes. In that case, gains over eICIC-only are observed, especially for large cluster sizes. Authors in [24] propose centralized and decentralized CoMP CS schemes that utilize CSI $\mathrm{I}_{\text {lte }}^{\mathrm{R}-11}$ reports, in which muting is applied to one BS at a time. A BS is called muted if it does not transmit data on a specific time/frequency resource. It has been shown that under this muting condition, both centralized and decentralized schemes achieve the same performance, favoring the decentralized scheme due to the reduced information exchange. Moreover, in [25], the authors extend the cooperation scheme of [24], to introduce muting of more than one BS per scheduling decision in a larger network. A greedy CS algorithm is presented to solve the centralized problem, which yields limited additional gain with respect to the decentralized scheme with overlapping cooperation clusters. The coordination scheme of [25] consists in a greedy optimization procedure. It is therefore suboptimal and further investigation regarding the optimally achievable performance of coordination, in the case of $\mathrm{CSI}_{\text {lte }}^{\mathrm{R}-11}$ reports, has not been carried out. Additionally, the results are focused on macro-only networks, where the gains of cooperation are restricted due to similar interfering power levels experienced from multiple BSs.

Although the abovementioned works show that CoMP schemes enhance the user throughput with respect to a network operating without any cooperation, no detailed studies are carried out in order to establish the maximum achievable gains that CoMP schemes can offer in realistic network scenarios and under LTE-Advanced-specific CSI reports. In [26], it has been demonstrated from an analytical perspective that cooperative schemes have fundamentally limited gains. That is, even under the assumption of centralized coordination and ideal CSI in form of instantaneous channel coefficients, the cooperation gains are limited due to the residual interference from BSs outside of the cooperation area, the signaling overheads, and the finite nature of the time/frequency/space resources. In the paper at hand, such limits are investigated under practical conditions by means of system level evaluations. For that purpose, the CoMP CS problem is formulated and solved optimally for an LTE-Advanced downlink network with centralized architecture. The problem formulation is based on multiple CSI reports generated by the UEs and gathered by the central controller, which uses this information to determine the coordinated scheduling decisions for all connected BSs. The central controller then decides which BSs serve their connected UEs on a given time/frequency resource, and which BSs are muted in order to reduce the interference caused to the UEs served by the neighboring transmitting BSs. The main contributions of this work are summarized as follows:

- The CS problem, where BSs cooperate by muting time/frequency resources based on standardized $\mathrm{CSI}_{\mathrm{lte}}^{\mathrm{R}-11}$ reports, is formulated as an integer non-linear program (INLP).

- The non-linear CS with muting problem is reformulated into a computationally tractable equivalent integer linear program (ILP), which enjoys of low computational complexity and can be efficiently solved by commercial solvers.

- A configurable heuristic algorithm is proposed as an extension to the greedy algorithm in [25], which achieves an excellent trade-off between performance and computational complexity. 


\section{System model}

A cellular network is considered as illustrated in Fig. 1, where a cooperation cluster consisting of $M \mathrm{BSs}$, operating in frequency division duplexing (FDD) mode, serves $N$ UEs in the downlink. Orthogonal frequency division multiple access (OFDMA) is assumed with frequency reuse one, where at each transmission time, all BSs can make use of the same $L$ Physical Resource Blocks (PRBs) for transmission. Thus, inter-cell interference affects the UEs, especially at the cell-edge. The operation of the cooperation cluster is managed by a central controller with backhaul connectivity to all $M$ BSs. In the following, the sets of indices $\mathcal{M}=\{1, \ldots, M\}, \mathcal{N}=\{1, \ldots, N\}$, and $\mathcal{L}=\{1, \ldots, L\}$ are used to address the BSs, UEs, and PRBs, respectively.

The received power at $\mathrm{UE} n \in \mathcal{N}$, from BS $m \in \mathcal{M}$, on PRB $l \in \mathcal{L}$, is denoted as $p_{n, m, l}$. Hence, for single-inputsingle-output (SISO) transmission,

$$
p_{n, m, l}=\left|g_{n} h_{n, m, l}\right|^{2} \phi_{m, l},
$$

where $\phi_{m, l}$ corresponds to the transmit power of BS $m$ on PRB $l$, the complex coefficient $h_{n, m, l}$ represents the amplitude gain of the downlink channel between BS $m$ and UE $n$ on PRB $l$, and $g_{n}$ is the receiver amplitude processing gain. In (1), the transmitted symbols are assumed to exhibit unit average transmit power. When summing over all PRBs, the total received power at $\mathrm{UE} n$ from $\mathrm{BS} m$ is obtained as

$$
p_{n, m}=\sum_{l=1}^{L} p_{n, m, l} \text {. }
$$

Generally, the serving BS for UE $n \in \mathcal{N}$ is selected as the BS from which the highest total received power is obtained, as defined in (2). In the case of heterogeneous networks, in order to achieve load balancing and cell-splitting gains, techniques like cell range expansion are applied where the UEs are instructed to add a constant off-set in the computation of the total received power of the small cells [27, 28]. In this paper, both homogeneous macro-only and heterogeneous networks are studied, where for the latter case, cell range expansion is applied. The $N \times M$ connection matrix $\mathbf{C}$ is defined, with elements

$$
c_{n, m}=\left\{\begin{array}{l}
1 \text { if UE } n \text { is served by BS } m \in \mathcal{M} \\
0 \text { otherwise, }
\end{array}\right.
$$

characterizing the serving conditions between BSs and UEs, where it is assumed that only one BS serves UE $n$ over all PRBs. It is further assumed, for simplicity, that the UEs are quasi-static, such that no handover procedures are triggered between the BSs. Therefore, the connection matrix $\mathbf{C}$ is assumed to be constant during the considered operation time.

The set of indices of BSs within the cooperation cluster that interfere with $\mathrm{UE} n \in \mathcal{N}$ is defined as $\mathcal{I}_{n}=\left\{m \mid c_{n, m}=0, \forall m \in \mathcal{M}\right\}$, with cardinality $\left|\mathcal{I}_{n}\right|=M-1$. Moreover, since UE $n$ experiences different interfering power levels from the $\mathcal{I}_{n}$ interfering BSs, the set $\mathcal{I}_{n}^{\prime} \subseteq \mathcal{I}_{n}$ of indices of the $M^{\prime}$ strongest interfering BSs of UE $n$, is defined such that $\left|\mathcal{I}_{n}^{\prime}\right|=M^{\prime}$. Therefore, the set $\mathcal{I}_{n}^{\prime}$ contains the indices of the $M^{\prime}$ interfering BSs with the highest total received power at UE $n$, as calculated in (2). The number of strongest interfering BSs is bounded as $0 \leq M^{\prime} \leq M-1$, where the sets $\mathcal{I}_{n}^{\prime}$ and $\mathcal{I}_{n}$ are identical, if



Fig. 1 Cooperation cluster of M BSs and N UEs in the downlink. The BSs are connected through the backhaul to a central controller 
$M^{\prime}=M-1$. The sets $\mathcal{I}_{n}$ and $\mathcal{I}_{n}^{\prime}$ apply for all PRBs in the reporting period.

Within the cooperation cluster, the BSs cooperate in the form of coordinated scheduling with muting, as previously mentioned in Section 1 . The central controller is then, in charge of managing the downlink transmissions of the BSs, where at each transmission time and on a per PRB basis, each BS can be requested to abstain from transmitting data. Hence, the interference caused to UEs located in neighboring BSs is reduced on the PRBs with muted BSs. Given the muting decision matrix, $\bar{\alpha}$, of dimensions $M \times L$ and elements

$$
\bar{\alpha}_{m, l}=\left\{\begin{array}{l}
1 \text { if BS } m \in \mathcal{M} \text { is muted on } \operatorname{PRB} l \in \mathcal{L} \\
0 \text { otherwise }
\end{array}\right.
$$

the signal-to-interference-plus-noise ratio (SINR) of UE $n \in \mathcal{N}$, served by BS $k \in \mathcal{M}$, on PRB $l$, is then defined as

$$
\gamma_{n, l}\left(\overline{\boldsymbol{\alpha}}_{l}\right)=\frac{\left(1-\bar{\alpha}_{k, l}\right) p_{n, k, l}}{I_{n, l}^{\mathrm{cc}}\left(\overline{\boldsymbol{\alpha}}_{l}\right)+I_{n, l}^{\mathrm{oc}}+\sigma^{2}} .
$$

The vector, $\overline{\boldsymbol{\alpha}}_{l}$, is equivalent to the $l$-th column of $\overline{\boldsymbol{\alpha}}$. The numerator corresponds to the average received power at UE $n$, from the serving BS $k$, on PRB $l$, as defined in (1). The first term in the denominator corresponds to the average inter-cell interference from the BSs within the cooperation cluster, with

$$
I_{n, l}^{\mathrm{cc}}\left(\overline{\boldsymbol{\alpha}}_{l}\right)=\sum_{m \in \mathcal{I}_{n}}\left(1-\bar{\alpha}_{m, l}\right) p_{n, m, l},
$$

$I_{n, l}^{\mathrm{oc}}$ is the average out-of-cluster interference, and $\sigma^{2}$ is the noise power assumed, without loss of generality, to be constant for all UEs over all PRBs. It is worth noting that the out-of-cluster interference $I_{n, l}^{\mathrm{oc}}$ is assumed to be independent of the muting decision matrix $\overline{\boldsymbol{\alpha}}$, since the central controller is not aware of the muting decisions made by the BSs outside of the cooperation cluster.

The achievable data rate of UE $n \in \mathcal{N}$ on PRB $l \in \mathcal{L}$ is modeled as a function of the UE's SINR. Hence,

$$
r_{n, l}=f\left(\gamma_{n, l}\right),
$$

where $f\left(\gamma_{n, l}\right)$ denotes a mapping from the SINR of UE $n$ on PRB $l$, to the achievable data rate.

In the literature, it is common to assume that the central controller has perfect CSI knowledge, in form of the instantaneous channel coefficients, $h_{n, m, l}$, as introduced in (1). Thus, the computation of the SINR, $\gamma_{n, l}\left(\overline{\boldsymbol{\alpha}}_{l}\right)$, and the achievable data rates, $r_{n, l}$, as defined in (5) and (7), respectively, is carried out in a straightforward manner for any possible muting decision $\overline{\boldsymbol{\alpha}}$. However, in practical conditions such as in LTE-Advanced networks, the CSI is typically available in form of achievable data rate measurement reports, i.e., CSI reports, which contain average information of multiple time/frequency/space resources for a subset of possible muting decisions $\bar{\alpha}$, as defined in (4). Thus, the processing and signaling overhead is reduced, at the expense of limited CSI knowledge for the CoMP CS scheme.

\section{CSI reporting for LTE-Advanced CoMP CS}

To enable opportunistic scheduling and CoMP operation in LTE-Advanced, CSI estimation is supported by the transmission of CSI reference signals (CSI-RSs) from the BSs $[29,30]$. One main feature of the CSI-RSs is the possibility to configure muted CSI-RSs, i.e., CSI-RSs with zero transmission power, enabling the UEs to estimate CSI from specific neighboring BSs without interference from the serving BS. Therefore, the UEs can generate multiple $\mathrm{CSI}_{\text {lte }}^{\mathrm{R}-11}$ reports that reflect different serving and interfering conditions in the network [20, 21]. These $\mathrm{CSI}_{\text {lte }}^{\mathrm{R}-11}$ reports are typically composed of a channel quality indicator (CQI), a precoding matrix index (PMI), and a rank indication (RI) [31]. The CQI reflects the measured/estimated SINR and the corresponding achievable data rate of a UE, when assuming a downlink transmission of rank indicated by the RI and transmit precoding vector taken from a finite-length codebook as indexed by the PMI.

For a cooperation cluster with $M$ BSs, a total of $J=2^{M}-1$ muting decisions can be made per PRB $l \in \mathcal{L}$. In this work, the practical case is considered, where the CS operation is managed by the central controller based on the $\mathrm{CSI}_{\text {lte }}^{\mathrm{R}-11}$ reports provided by the UEs. Since the SINRs and achievable data rates of UE $n \in \mathcal{N}$, as defined in (5) and (7), respectively, are dominated by its strongest interfering BSs [24], in the following, it is assumed that the UEs generate a total of $J^{\prime}=2^{M^{\prime}} \operatorname{CSI}_{\text {lte }}^{\mathrm{R}-11}$ reports per PRB, with $J^{\prime}<J$. Then, each $\mathrm{CSI}_{\text {lte }}^{\mathrm{R}-11}$ report only considers the $M^{\prime}$ strongest interfering BSs of UE $n$, as described by the set $\mathcal{I}_{n}^{\prime}$, introduced in Section 2.

Each of the $J^{\prime} \mathrm{CSI}_{\text {lte }}^{\mathrm{R}-11}$ reports, which are generated by $\mathrm{UE} n \in \mathcal{N}$ on PRB $l \in \mathcal{L}$, reflects a unique interference scenario for its strongest interfering BSs. More specifically, the interference scenario $j \in \mathcal{J}^{\prime}$, with $\mathcal{J}^{\prime}=\left\{1, \ldots, J^{\prime}\right\}$, is characterized by the muting indicator set $\mathcal{J}_{n, j}$, which contains the indices of the (strongest) interfering BSs considered to be muted in the $j$-th $\mathrm{CSI}_{\text {lte }}^{\mathrm{R}-11}$ report of UE $n$. Hence, the set $\mathcal{J}_{n}=\mathbb{P}\left(\mathcal{I}_{n}^{\prime}\right)$ contains all $J^{\prime}$ muting indicator sets for UE $n$, with $\mathbb{P}(\cdot)$ denoting the set of all subsets of $\mathcal{I}_{n}^{\prime}$. The set $\mathcal{J}_{n}$ is common to all PRBs. From the muting indicator set, $\mathcal{J}_{n, j}$, the muting pattern of the $j$-th $\operatorname{CSI}_{\text {lte }}^{\mathrm{R}-11}$ report of UE $n$, on PRB $l$, is defined as

$$
\alpha_{n, m^{\prime}, l, j}=\left\{\begin{array}{l}
1 \text { if } m^{\prime} \in \mathcal{J}_{n, j} \text { on PRB } l \\
0 \text { otherwise, }
\end{array} \forall m^{\prime} \in \mathcal{I}_{n}^{\prime},\right.
$$

i.e., $\alpha_{n, m^{\prime}, l, j}=1$, if the (strongest) interfering BS $m^{\prime}$, is muted on PRB $l$, under interference scenario $j$. The definition in (8) considers only the set of strongest interfering 
BSs of UE $n$, i.e., $\mathcal{I}_{n}^{\prime}$. Therefore, a constant muting state of the remaining BSs in the cooperation cluster is required, for all $\mathcal{J}^{\prime}$ interference scenarios. In the following, it is assumed without loss of generality that $\alpha_{n, m, l, j}=0$, $\forall m \notin \mathcal{I}_{n}^{\prime}, \forall j \in \mathcal{J}^{\prime}$. Although the definition of the muting pattern in (8) is similar to the definition of the muting decision in (4), the two concepts are different. The muting pattern describes the assumed muting conditions during the generation of the $\operatorname{CSI}_{\text {lte }}^{\mathrm{R}-11}$ reports for the different interference scenarios, while the muting decision is imposed by the central controller, to the BSs within the cooperation cluster, as the result of the implementation of the CS with muting scheme.

For the generation of the $\mathrm{CSI}_{\text {lte }}^{\mathrm{R}-11}$ reports, $\mathrm{UE} n \in \mathcal{N}$ calculates the SINR and the achievable data rates, on PRB $l \in \mathcal{L}$ under interference scenario $j \in \mathcal{J}^{\prime}$. Therefore, similar to (5), the SINR of UE $n$ on PRB $l$, under interference scenario $j$, is defined as

$$
\gamma_{n, l, j}\left(\alpha_{n, m^{\prime}, l, j}\right)=\frac{p_{n, k, l}}{I_{n, l, j}^{\mathrm{si}}\left(\alpha_{n, m^{\prime}, l, j}\right)+I_{n, l}^{\mathrm{wi}}+I_{n, l}^{\mathrm{oc}}+\sigma^{2}},
$$

where the first term in the denominator of (5) has been decomposed into two terms corresponding to the interference from the strongest interfering BSs of UE $n$, i.e., $I_{n, l, j}^{\mathrm{si}}\left(\alpha_{n, m^{\prime}, l, j}\right)$, and the interference from the remaining (weakest) interfering BSs of UE $n$, denoted by $I_{n, l}^{\mathrm{wi}}$. The interference from the strongest interfering BSs that can cooperate to improve the SINR of UE $n$ depends on interference scenario $j$, and thus, the muting pattern, as

$$
I_{n, l, j}^{\mathrm{si}}\left(\alpha_{n, m^{\prime}, l, j}\right)=\sum_{m^{\prime} \in \mathcal{I}_{n}^{\prime}}\left(1-\alpha_{n, m^{\prime}, l, j}\right) p_{n, m^{\prime}, l} .
$$

On the other hand, the interference from the weakest interfering BSs of UE $n$ is assumed to be constant and independent of the possible muting decisions, with

$$
I_{n, l}^{\mathrm{wi}}=\sum_{m \in \mathcal{I}_{n} \backslash \mathcal{I}_{n}^{\prime}} p_{n, m, l} .
$$

Furthermore, the out-of-cluster interference and the noise powers are also assumed to be constant terms among all the $J^{\prime}$ interfering scenarios considered in the $\mathrm{CSI}_{\text {lte }}^{\mathrm{R}-11}$ reports.

To complete the information for the $\operatorname{CSI}_{\text {lte }}^{\mathrm{R}-11}$ reports, $r_{n, l, j}$ denotes the achievable data rate of UE $n \in \mathcal{N}$, on PRB $l \in \mathcal{L}$, under interference scenario $j \in \mathcal{J}^{\prime}$. The calculation of $r_{n, l, j}$ follows the definition in (7), as a function of $\gamma_{n, l, j}$ given by (9). In this work, it is assumed that the UEs reflect their achievable data rates for the interference scenarios $j \in \mathcal{J}^{\prime}$, into the CQI values to be reported in the CSI $\mathrm{Ilte}_{\text {lte }}^{\mathrm{R}}$ reports. Additionally, it is further assumed that the BSs can translate the CQI values from the $\mathrm{CSI}_{\text {lte }}^{\mathrm{R}-11}$ reports, into the corresponding achievable data rates in order to support the cooperative scheduling process. Therefore, in the following, the description is focused on the achievable data rates extracted from the $\mathrm{CSI}_{\mathrm{lte}}^{\mathrm{R}-11}$ reports.

By inspecting (9) and (10), the SINR of UE $n \in \mathcal{N}$, on PRB $l \in \mathcal{L}$, increases when muting additional interfering BSs. Correspondingly, the achievable data rate of UE $n$, on PRB $l$, increases or remains constant with the increased SINR, if the function $f\left(\gamma_{n, l, j}\right)$ is non-decreasing.

Example 1 In an exemplary network with a cooperation cluster of $M=4 B S s$ and a total of $M^{\prime}=2$ strongest interfering BSs per $U E, U E n \in \mathcal{N}$ selects $B S 1$ and $B S 2$ for cooperation, such that $\mathcal{I}_{n}^{\prime}=\{1,2\}$. Thus, UE $n$ generates $J^{\prime}=4$ CSI lte $_{\text {lt }}^{\text {-11 }}$ reports on $P R B l \in \mathcal{L}$, as summarized in Table 1, with $r_{n, l, 2} \geq r_{n, l, 3}, r_{n, l, 4} \geq r_{n, l, 1}$.

\section{CS with muting}

\subsection{Proposed INLP-problem formulation}

At the central controller, the $\mathrm{CSI}_{\mathrm{lte}}^{\mathrm{R}-11}$ reports, generated by the UEs and forwarded by the BSs, are used in order to compute the CS decision. The CS decision consists of two main components, namely, a scheduling decision that assigns PRBs to UEs, and a muting decision that mutes BSs on particular PRBs. The matrix variable $\overline{\mathbf{S}}$ of dimensions $N \times L$ and elements

$$
\bar{s}_{n, l}=\left\{\begin{array}{l}
1 \text { if } \operatorname{PRB} l \in \mathcal{L} \text { is assigned to UE } n \in \mathcal{N} \\
0 \text { otherwise }
\end{array}\right.
$$

is used to denote the scheduling decision for all UEs on each PRB $l$, while the $M \times L$ matrix variable $\overline{\boldsymbol{\alpha}}$, with elements as introduced in (4), refers to the muting decision for all BSs on each PRB $l$. In the following, an INLP is proposed, to carry out joint BS muting and UE scheduling in a coordinated network.

Typically, the schedulers in mobile communications pursue a trade-off between user throughput and fairness. For that purpose, opportunistic scheduling is applied such as in the case of the Proportional Fair (PF) scheduler $[32,33]$. The objective of the PF scheduler is to maximize

\begin{tabular}{|c|c|c|c|}
\hline $\begin{array}{l}\text { Int. scenario } \\
\left(j \in \mathcal{J}^{\prime}\right)\end{array}$ & $\begin{array}{l}\text { Mut. ind. } \\
\left(\mathcal{J}_{n, j}\right)\end{array}$ & $\begin{array}{l}\text { Mut. pattern } \\
\left(\alpha_{n, l}\right)\end{array}$ & $\begin{array}{l}\text { Achiev. } \\
\text { data rate }\end{array}$ \\
\hline 1 & $\{\varnothing\}$ & {$[0,0,0,0]$} & $r_{n, 1,1}$ \\
\hline 2 & $\{1,2\}$ & {$[1,1,0,0]$} & $r_{n, l, 2}$ \\
\hline 3 & $\{1\}$ & {$[1,0,0,0]$} & $r_{n, l, 3}$ \\
\hline 4 & $\{2\}$ & {$[0,1,0,0]$} & $r_{n, l, 4}$ \\
\hline
\end{tabular}
the sum, over all UEs, of the PF metrics given by

$$
\Omega_{n}=\frac{r_{n}}{R_{n}} \forall n \in \mathcal{N},
$$


where the ratio between the total instantaneous achievable data rate and the average user throughput over time, denoted by $r_{n}$ and $R_{n}$, respectively, of UE $n \in \mathcal{N}$ is considered. The total instantaneous achievable data rate of UE $n$ is calculated as

$$
r_{n}=g\left(r_{n, l, j}, \overline{\boldsymbol{s}}_{n}, \overline{\boldsymbol{\alpha}}\right) \forall l \in \mathcal{L}, \forall j \in \mathcal{J}^{\prime},
$$

where $g(\cdot)$ corresponds to a function of the achievable data rates of UE $n$, denoted by $r_{n, l, j}$, over the PRBs assigned to UE $n$, as described by the $n$-th row of $\overline{\mathbf{S}}$, denoted by $\overline{\boldsymbol{s}}_{n}$, and the muting decision matrix $\overline{\boldsymbol{\alpha}}$.

The LTE-Advanced CS with muting problem can be formulated as the following INLP

$$
\begin{aligned}
& \max _{\{\overline{\mathbf{s}}, \bar{\alpha}\}} \sum_{n \in \mathcal{N}} \Omega_{n} \\
& \text { s.t. } \\
& \bar{\alpha}_{m, l}+\sum_{n \in \mathcal{N}} c_{n, m} \bar{s}_{n, l} \leq 1 \forall m \in \mathcal{M}, \forall l \in \mathcal{L}, \\
& r_{n}=\sum_{l \in \mathcal{L}} \rho\left(\boldsymbol{r}_{n, l}, \overline{\boldsymbol{\alpha}}_{l}, \mathcal{I}_{n}^{\prime}\right) \bar{s}_{n, l} \forall n \in \mathcal{N}, \\
& \bar{s}_{n, l} \in\{0,1\} \forall n \in \mathcal{N}, \forall l \in \mathcal{L}, \\
& \bar{\alpha}_{m, l} \in\{0,1\} \forall m \in \mathcal{M}, \forall l \in \mathcal{L},
\end{aligned}
$$

where the objective in (15a) is to maximize the sum of the PF metrics over all UEs, with the PF metric of UE $n \in \mathcal{N}$ calculated as in (13). The constraints in (15b) link the scheduling decision $\overline{\mathbf{S}}$ with the muting decision $\overline{\boldsymbol{\alpha}}$. If BS $m \in \mathcal{M}$ is muted on PRB $l \in \mathcal{L}$, then PRB $l$ cannot be assigned to any UE $n$ connected to BS $m$. Thus, if $\bar{\alpha}_{m, l}=1$ in (15b), for BS $m$, the second term on the left-handside must be equal to zero, which is true in either of the following cases, with the connection indicator $c_{n, m}$ given by (3):

- No UEs are connected to BS $m$, i.e., $c_{n, m}=0, \forall n \in \mathcal{N}$.

- PRB $l$ is not assigned to any UE served by BS $m$, i.e., $\bar{s}_{n, l}=0, \forall n \in \mathcal{N}$ such that $c_{n, m}=1$.

Furthermore, in the case that BS $m$ is not muted on PRB $l$, i.e., $\bar{\alpha}_{m, l}=0$, the constraints in (15b) ensure that single user transmissions are carried out, where each BS is allowed to schedule a maximum of one UE per PRB. Additionally, the total instantaneous achievable data rate of UE $n$, denoted by $r_{n}$ as introduced in (14), is calculated in (15c), with

$$
g\left(r_{n, l, j}, \overline{\boldsymbol{s}}_{n}, \overline{\boldsymbol{\alpha}}\right)=\sum_{l \in \mathcal{L}} \rho\left(\boldsymbol{r}_{n, l}, \overline{\boldsymbol{\alpha}}_{l}, \mathcal{I}_{n}^{\prime}\right) \bar{s}_{n, l} \forall n \in \mathcal{N} .
$$

In (16), $\rho\left(\boldsymbol{r}_{n, l}, \overline{\boldsymbol{\alpha}}_{l}, \mathcal{I}_{n}^{\prime}\right)$ is a lookup table function that selects the achievable data rate of UE $n$, on PRB $l$, based on the muting decision $\overline{\boldsymbol{\alpha}}_{l}$ of the strongest interfering BSs of UE $n$, as indexed by $\mathcal{I}_{n}^{\prime}$. The lookup table function $\rho(\cdot)$ selects the achievable data rate from the $J^{\prime} \times 1$ vector, $\boldsymbol{r}_{n, l}$, with elements $r_{n, l, j}, \forall j \in \mathcal{J}^{\prime}$, obtained from the CSI $\mathrm{lte}_{\mathrm{lte}}^{\mathrm{R}-11}$ reports of UE $n$, on PRB $l$.

Example 2 Based on the Table 1 from Example 1, with $J^{\prime}=4 \mathrm{CSI}_{\text {lte }}^{R-11}$ reports, the lookup table function for $U E$ $n \in \mathcal{N}$, on PRB $l \in \mathcal{L}$, provides the results as in Table 2. Note that the value of $\rho(\cdot)$ does not depend on the muting decision of the remaining $B S$ s.

Due to the utilization of the $J^{\prime} \operatorname{CSI}_{\text {lte }}^{\mathrm{R}-11}$ reports, the achievable data rate of UE $n \in \mathcal{N}$, on PRB $l \in \mathcal{L}$, under interference scenario $j \in \mathcal{J}^{\prime}$, is constant in the problem formulation and limited to the set of reported muting patterns. In Section 3, it has been mentioned that the achievable data rate of UE $n$, on PRB $l$, under interference scenario $j$ depends on the function $f\left(\gamma_{n, l, j}\right)$. If $f(\cdot)$ is piecewise non-decreasing, it follows that additional (strongest) interfering BSs are only muted if the achievable data rate of UE $n$ is increased.

Moreover, the scheduling and muting matrix variables, $\overline{\mathbf{S}}$ and $\overline{\boldsymbol{\alpha}}$, are binary as described by the constraints in (15d) and (15e), respectively.

The following remarks summarize the main characteristics of the LTE-Advanced CS with muting problem formulation in (15).

- As mentioned in Section 3, given $M^{\prime}$ strongest interfering BSs per UE $n \in \mathcal{N}$, a total of $J^{\prime}=2^{M^{\prime}}$ interfering scenarios per UE $n$ are available. Hence, two special cases of the problem formulation are observed:

(i) If $M^{\prime}=0$, each UE $n$ generates one $\mathrm{CSI}_{\text {lte }}^{\mathrm{R}-11}$ report under the assumption of no BS muting. At the central controller, the CS with muting problem formulation becomes a PF scheduler without any cooperation.

(ii) If $M^{\prime}=M-1$, all the interfering BSs within the cooperation cluster can be muted to improve the performance of any UE, on each PRB $l \in \mathcal{L}$. If the network size is large, finding the solution while assuming cooperation of all interfering BSs for all UEs approximates an exhaustive search.

- Due to the combinatorial nature of the problem formulation, it is classified as non-deterministic polynomial-time (NP)-hard.

Table 2 Lookup table function $\rho\left(\boldsymbol{r}_{n, l}, \bar{\alpha}_{l}, \mathcal{I}_{n}^{\prime}\right)$ for UE $n$, on PRB I, with $M^{\prime}=2$

\begin{tabular}{ll}
\hline $\bar{\alpha}_{m, l}, \forall m \in \mathcal{I}_{n}^{\prime}$ & $\rho\left(\boldsymbol{r}_{n, l}, \bar{\alpha}_{l,} \mathcal{I}_{n}^{\prime}\right)$ \\
\hline$[0,0]$ & $r_{n, 1,1}$ \\
{$[0,1]$} & $r_{n, l, 4}$ \\
{$[1,0]$} & $r_{n, l, 3}$ \\
{$[1,1]$} & $r_{n, l, 2}$ \\
\hline
\end{tabular}


- The problem is non-linear because of the relation between the muting and the scheduling decision variables, $\overline{\boldsymbol{\alpha}}_{l}$ and $\bar{s}_{n, l}$, respectively, in the constraints in $(15 c)$.

Although the number of reported interference scenarios $J^{\prime}=2^{M^{\prime}}$ can be limited by selecting a small value $M^{\prime}$ of (strongest) interfering BSs per UE $n \in \mathcal{N}$, the CS with muting INLP formulation in (15) also depends on the number of UEs, i.e., $N$, and the number of PRBs, denoted by $L$. For certain network scenarios, $N$ and $L$ can be large. Therefore, given the non-linear nature of the problem in (15), finding a solution with commercial solvers may either not be possible or inefficient in terms of computation time. In the following, separability, reducibility, and lifting concepts are used, in order to formulate parallel ILP sub-problems that scale better with the network size.

\subsection{Proposed ILP_-parallelized sub-problem formulation 4.2.1 Separability}

When analyzing the objective function described by (15a), the total PF metric corresponds to the sum of the individual PF metrics for all UEs. Furthermore, at each UE $n \in \mathcal{N}$, it is assumed that the total instantaneous achievable data rate is equivalent to the linear combination of the decoupled achievable data rates per scheduled PRBs, as given by (15c). Therefore, it is possible to separate the CS with muting problem in (15), into $L$ independent subproblems, corresponding to the scheduling decision of one PRB each. By performing this parallelization, the computation time is reduced without affecting the quality of the solution, i.e., the solution of the parallelized CS with muting problem remains optimal.

\subsubsection{Reducibility}

It is expected that some of the UEs, connected to a common BS $m \in \mathcal{M}$, share one or more strongest interfering BSs. From a BS perspective, the set

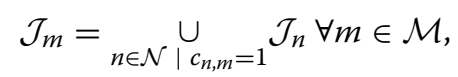

contains all the unique muting indicator sets, associated to its connected UEs. Similar to the set $\mathcal{J}_{n}, \mathcal{J}_{m}$ is common to all PRBs in the reporting period. The number of unique muting indicator sets for BS $m$, i.e., $J_{m}^{\prime}=\left|\mathcal{J}_{m}\right|$, depends on the number of UEs connected to BS $m$ and the maximum number $J^{\prime}$ of reported interference scenarios per UE, as introduced in Section 3. Thus, $J^{\prime} \leq J_{m}^{\prime} \leq$ $\sum_{n \in \mathcal{N}} c_{n, m} J^{\prime}$, where the lower bound corresponds to the case when all connected UEs are interfered by the same set of strongest interfering BSs, and the upper bound represents the case with all UEs having different strongest interfering BSs. For the unique muting indicator set $\mathcal{J}_{m, j^{\prime}}$, with $j^{\prime} \in \mathcal{J}_{m}^{\prime}=\left\{1, \ldots, J_{m}^{\prime}\right\}$, the set of indices of UEs, connected to BS $m$, with equal muting indicator set is defined as

$$
\begin{aligned}
\mathcal{N}_{m, j^{\prime}}=\left\{n \in \mathcal{N} \mid c_{n, m}=1, \mathcal{J}_{m, j^{\prime}} \subsetneq \mathcal{J}_{n},\right. \\
\left.\forall m \in \mathcal{M}, \forall j^{\prime} \in \mathcal{J}_{m}^{\prime}\right\} .
\end{aligned}
$$

Based on the definitions in (17) and (18), and taking into account that single user transmissions take place per BS, it is sufficient that each BS $m \in \mathcal{M}$ forwards to the central controller, the $\mathrm{CSI}_{\text {lte }}^{\mathrm{R}-11}$ reports related to one UE per unique muting indicator set $\mathcal{J}_{m, j^{\prime}}, \forall j^{\prime} \in \mathcal{J}_{m}^{\prime}$, on PRB $l \in \mathcal{L}$, instead of the $\mathrm{CSI}_{\text {lte }}^{\mathrm{R}-11}$ reports from all connected UEs. The set of indices of UEs connected to BS $m$ that maximize the PF metric, in at least one of the unique muting indicator sets indexed by $j^{\prime} \in \mathcal{J}_{m}^{\prime}$, on PRB $l$, is defined as

$$
\begin{aligned}
\mathcal{N}_{m, l}^{\prime}= & \left\{\hat{n} \mid \exists j: \hat{n}=\underset{n \in \mathcal{N}_{m, j^{\prime}}}{\arg \max } \Omega_{n, l, j},\right. \\
& \left.\forall j^{\prime} \in \mathcal{J}_{m}^{\prime}, \forall j \in \mathcal{J}^{\prime} \mid \mathcal{J}_{n, j}=\mathcal{J}_{m, j^{\prime}}\right\} .
\end{aligned}
$$

The cardinality of the set $\mathcal{N}_{m, l}^{\prime}$, denoted as $\left|\mathcal{N}_{m, l}^{\prime}\right|$, is bounded as $1 \leq\left|\mathcal{N}_{m, l}^{\prime}\right| \leq \sum_{n \in \mathcal{N}} c_{n, m}$, where the lower bound implies that only one UE provides the maximum PF metric, among all unique muting indicator sets on PRB $l$. The upper bound corresponds to the case when each UE reports different muting indicator sets with respect to the other UEs connected to BS $m$.

At the central controller, all the achievable data rates, $r_{n, l, j}, \forall n \in \mathcal{N}_{m, l}^{\prime}, \forall m \in \mathcal{M}, \forall l \in \mathcal{L}, \forall j \in \mathcal{J}^{\prime}$, are per definition set to zero, for the interference scenarios where UE $n$ does not provide the maximum PF metric, among the UEs connected to the same BS $m$. The set $\mathcal{N}_{l}^{\prime}=\underset{m \in \mathcal{M}}{\cup} \mathcal{N}_{m, l}^{\prime}$ is used to denote the indices of UEs to be considered in the reformulated ILP, on PRB $l$. The cardinality of the set $\mathcal{N}_{l}^{\prime}$ is described as $M \leq\left|\mathcal{N}_{l}^{\prime}\right| \leq N$. In the special case of $M^{\prime}=0$, all UEs report only one interference scenario where no cooperative interfering BS is muted, and thus, $\left|\mathcal{N}_{l}^{\prime}\right|=M$.

\subsubsection{Lifting}

In order to linearize the constraints in (15c), a variable transformation is introduced based on the lifting technique [34]. A new coordinated decision variable is defined containing both, the scheduling and the muting decisions, as

$$
s_{n, l, j}=\left\{\begin{array}{c}
1 \text { if PRB } l \in \mathcal{L} \text { is assigned to } \mathrm{UE} n \in \mathcal{N} \\
\text { under interference scenario } j \in \mathcal{J}^{\prime} \\
0 \text { otherwise. }
\end{array}\right.
$$

The new decision variable, $s_{n, l, j}$, is related to the muting and scheduling decisions in (4) and (12), respectively, as

$$
\begin{aligned}
s_{n, l, j}=1 \Leftrightarrow & \bar{s}_{n, l}=1 \wedge \bar{\alpha}_{m, l}=1 \\
& \forall n \in \mathcal{N}, \forall l \in \mathcal{L}, \forall j \in \mathcal{J}^{\prime}, \forall m \in \mathcal{J}_{n, j},
\end{aligned}
$$


with $\wedge$ denoting the logical and operator. Hence, the nonlinear constraints in $(15 \mathrm{c})$ reduce to a linear combination of the achievable data rates, i.e., $r_{n, l, j}$, and the new decision variable, $s_{n, l, j}$.

\subsubsection{Problem reformulation}

Using the above described concepts of separability, reducibility, and lifting, the CS with muting INLP formulation in (15) can be reformulated as an ILP, which can be efficiently solved by commercial solvers. Hence, with the set $\mathcal{N}_{l}^{\prime}$ and defining the binary decision variable $\mathbf{S}_{l}$ to have dimensions $\left|\mathcal{N}_{l}^{\prime}\right| \times J^{\prime}$, the sub-problem formulation for PRB $l \in \mathcal{L}$ is

$$
\begin{aligned}
& \max _{\left\{\mathbf{S}_{l}\right\}} \sum_{n \in \mathcal{N}_{l}^{\prime}} \Omega_{n, l} \\
& \text { s.t. } \\
& s_{n, l, j}+\sum_{k \in \mathcal{N}_{l}^{\prime}} \sum_{i \in \mathcal{J}^{\prime}} c_{k, m} s_{k, l, i} \leq 1 \\
& \quad \forall n \in \mathcal{N}_{l}^{\prime}, \forall j \in \mathcal{J}^{\prime}, \forall m \in \mathcal{J}_{n, j}, \\
& \sum_{n \in \mathcal{N}_{l}^{\prime}} \sum_{j \in \mathcal{J}^{\prime}} c_{n, m} s_{n, l, j} \leq 1 \\
& \quad \forall m \in \mathcal{M} \backslash \cup_{n \in \mathcal{N}_{l}^{\prime}} \mathcal{I}_{n}^{\prime}, \\
& r_{n, l}=\sum_{j \in \mathcal{J}^{\prime}} r_{n, l, j} s_{n, l, j} \forall n \in \mathcal{N}_{l}^{\prime}, \\
& s_{n, l, j}=0 \forall n \in \mathcal{N}_{l}^{\prime}, \forall j \in \mathcal{J}^{\prime} \mid r_{n, l, j}=0, \\
& s_{n, l, j} \in\{0,1\} \forall n \in \mathcal{N}_{l}^{\prime}, \forall j \in \mathcal{J}^{\prime},
\end{aligned}
$$

where the objective in (22a) is to maximize the sum of the PF metric over all UEs. The constraints in (22b) restrict the scheduling decisions of the strongest interfering BSs of UE $n \in \mathcal{N}_{l}^{\prime}$, i.e., $\forall m \in \mathcal{J}_{n, j}$, in order to agree with the muting state considered in the interference scenario $j \in \mathcal{J}^{\prime}$. If PRB $l$ is assigned to UE $n$, under the condition of muting the (strongest) interfering BSs indexed by the set $\mathcal{J}_{n, j} \in \mathcal{J}_{n}$, then no other UE connected to the muted BSs can be simultaneously scheduled on the same PRB $l$. Thus, if $s_{n, l, j}=1$ in (22b), the second term on the left-handside must be equal to zero. Furthermore, in the case that $s_{n, l, j}=0$, the constraints in (22b) ensure that single user transmissions are carried out, where each BS $m \in \mathcal{J}_{n, j}$ is allowed to schedule a maximum of one UE per PRB, over all possible interference scenarios $j \in \mathcal{J}^{\prime}$. Since it is possible that specific BSs, within the cooperation cluster, do not belong to the set of strongest interfering BSs of any $\mathrm{UE}$, the constraints in (22c) complement the restriction on the single user transmissions from (22b). Additionally, the total instantaneous achievable data rate of UE $n$, on PRB $l$, denoted by $r_{n, l}$, is calculated in (22d) as the achievable data rate for the selected interference scenario $j$, as defined by the coordinated decision variable $s_{n, l, j}$. It is worth noting that there is a one-to-one mapping between $r_{n, l, j}$ and $s_{n, l, j}$, thus, there is no requirement for a lookup table function as used in (16). Furthermore, the constraints in (22e) are incorporated as a preprocessing step to ensure that no PRB is scheduled to UEs for which a maximum PF metric for the corresponding interference scenario $j$ is not available. Finally, the coordinated decision variable $\mathbf{S}_{l}$ is binary as described by the constraints in (22f).

It can be easily proven that the problem formulations in (15) and (22) are equivalent. Furthermore, the proposed parallelized formulation in (22), reduces significantly the CS with muting problem complexity, allowing its application even for large-size networks.

\subsection{Generalized greedy heuristic algorithm}

The greedy heuristic deflation algorithm in [25] (see algorithm in Section II) iteratively solves the CS with muting problem per PRB $l \in \mathcal{L}$, where at each iteration one BS is muted, corresponding to the $\mathrm{BS} m \in \mathcal{M}$ that, when muted, maximizes the sum of the PF metrics among all UEs on PRB $l$. The algorithm stops when muting any additional BS does not improve the sum of the PF metrics with respect to the previous iteration. There is no guarantee that the heuristic algorithm yields a globally optimal point because the quality of the scheduling decision depends directly on the gain achieved from muting one interfering $\mathrm{BS}$ at a time.

Given the abovementioned disadvantage of the CS with muting greedy heuristic algorithm from [25], an extension is proposed in this work, called generalized greedy heuristic algorithm, which trades off computational complexity with performance gains. The main difference, with respect to the algorithm in [25], is the evaluation of additional muting patterns per iteration, where for $\operatorname{PRB} l \in \mathcal{L}$, the set of muting indicators

$$
\hat{\mathcal{M}}=\bigcup_{\hat{m} \in\{1, \ldots, \tilde{m}\}}\left(\begin{array}{c}
\mathcal{M}^{\prime} \\
\hat{m}
\end{array}\right),
$$

defines the muting patterns to be evaluated. In (23), the binomial coefficients of the set $\mathcal{M}^{\prime}$, of possible muted BSs, are evaluated by selecting $\hat{m}$ BSs at a time. The configuration parameter $1 \leq \tilde{m} \leq M-1$, controls the complexity of the proposed generalized greedy heuristic algorithm by determining the muting patterns to be evaluated. If $\tilde{m}=1$, the generalized greedy heuristic algorithm reduces to the heuristic algorithm from [25]. In the case that $\tilde{m}=M-1$, the generalized greedy heuristic algorithm performs an exhaustive search.

\section{Simulation results}

In this section, extensive simulation results are presented to evaluate the performance of the CoMP CS schemes 
with respect to a PF scheduler without any cooperation, referred to as "non-coop. PFS". The proposed parallelized sub-problem formulation as presented in Section 4.2, labeled as "CS-ILP", is examined together with the greedy algorithm described in [25], denoted as "CS-GA", and the proposed generalized greedy algorithm of Section 4.3, labeled as "CS-GG". In the simulations, $M^{\prime}=2$ strongest interfering BSs per UE are considered.

\subsection{CS with muting-performance analysis}

In order to study the performance of the CS with muting schemes, Monte Carlo standalone simulations have been carried out, where the $\mathrm{CSI}_{\text {lte }}^{\mathrm{R}-11}$ reports are generated based on channels obtained from a 3rd Generation Partnership Project (3GPP) compliant system level simulator, as specified in [29, 35-37]. In each transmission time $t$, the average user throughput over time of $\mathrm{UE} n \in \mathcal{N}$, used in (13), is updated based on the scheduling decisions made at the previous transmission time $t-1$, as

$$
R_{n}(t)=\beta R_{n}(t-1)+(1-\beta) r_{n}(t-1),
$$

with $\beta=0.97$, denoting the forgetting factor parameter used to trade off user throughput and fairness [38]. The total instantaneous achievable data rate of UE $n$, at the previous transmission time, denoted by $r_{n}(t-1)$, is calculated as given by e.g., (22d).
Initially, the performance of the CS with muting algorithms in terms of average user throughput, is studied with respect to the data rates the users can achieve per symbol and to the noise power level considered in the calculation of these achievable data rates. In practical systems such as LTE-Advanced, finite modulation and coding schemes (MCSs) are used which restrict the achievable data rates per symbol to a given range $[39,40]$. For the current analysis, two cases are considered with respect to the maximum achievable data rate: (i) the MCS is unbounded, denoted as "Unb. MCS", and (ii) a maximum achievable data rate of $5.4 \mathrm{bits} / \mathrm{symbol}$ is used, referred to as "B. MCS". Similarly, there are two assumptions with respect to the noise power level, where in a first case, noise free decoding is assumed, denoted as "N.-less", which considers that $\sigma^{2}=\epsilon$, with $\epsilon$ arbitrarily small but larger than zero, and in a second, a typical receiver noise figure of $9 \mathrm{~dB}$ is considered, referred to as the "Noisy" case.

The cell-edge and the geometric mean of the user throughput are shown in Figs. 2 and 3, respectively, for a scenario with $M=3 \mathrm{BSs}, N=30$ UEs (ten UEs per BS) and $L=10$ PRBs. The cell-edge throughput describes the average user throughput of the cell-edge users and corresponds to the average throughput achieved by the worst $5 \%$ of the users. The usage of the geometric mean is proposed by the authors in [25] as a direct measure of the PF scheduler's objective function. The user throughputs achieved by the CS with muting schemes,

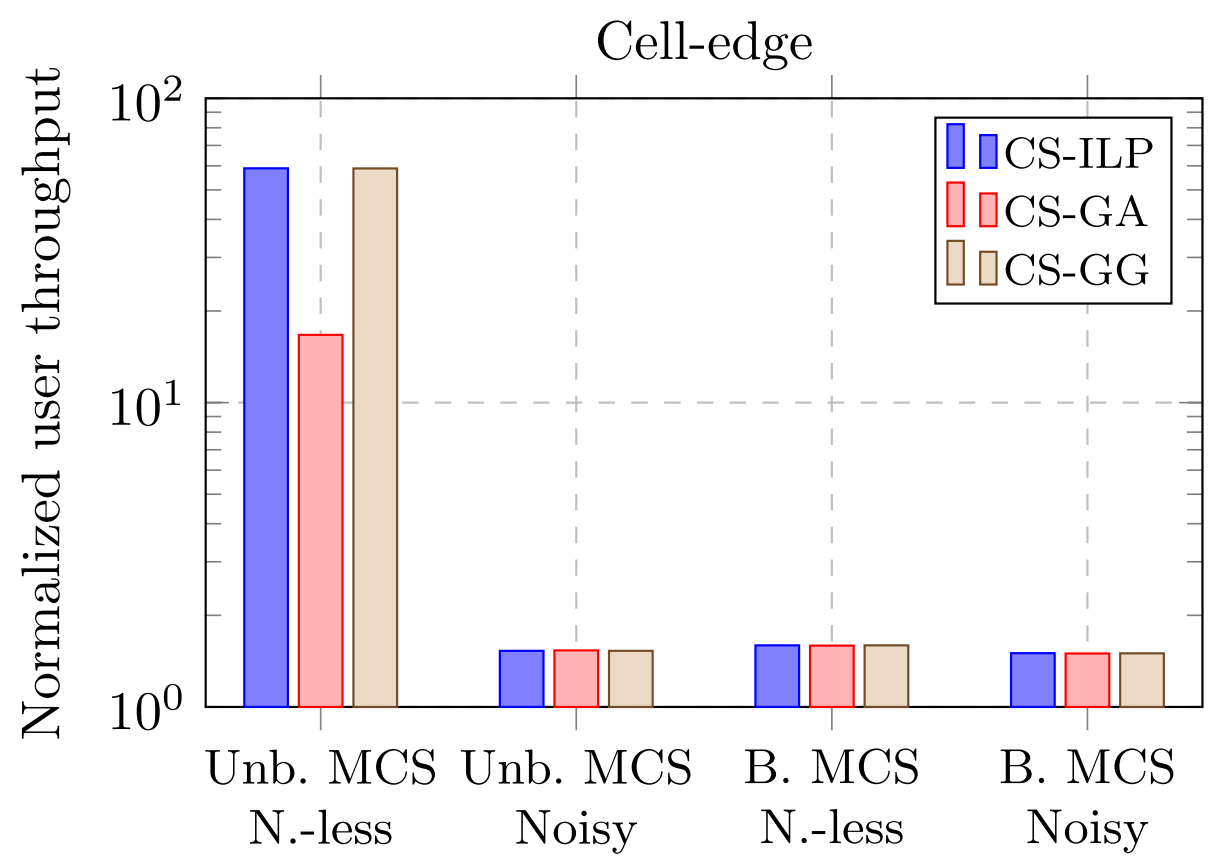

Fig. 2 Average cell-edge user throughput for the CoMP CS schemes, normalized with respect to the non-coop. PFS. Scenario with $M=3$ BSs, $N=30 \mathrm{UEs}, L=10 \mathrm{PRBs}$, and $M^{\prime}=2 \mathrm{BSs}$. Four cases with limitations on the maximum achievable data rate and the noise power level are considered. There is no out-of-cluster interference 


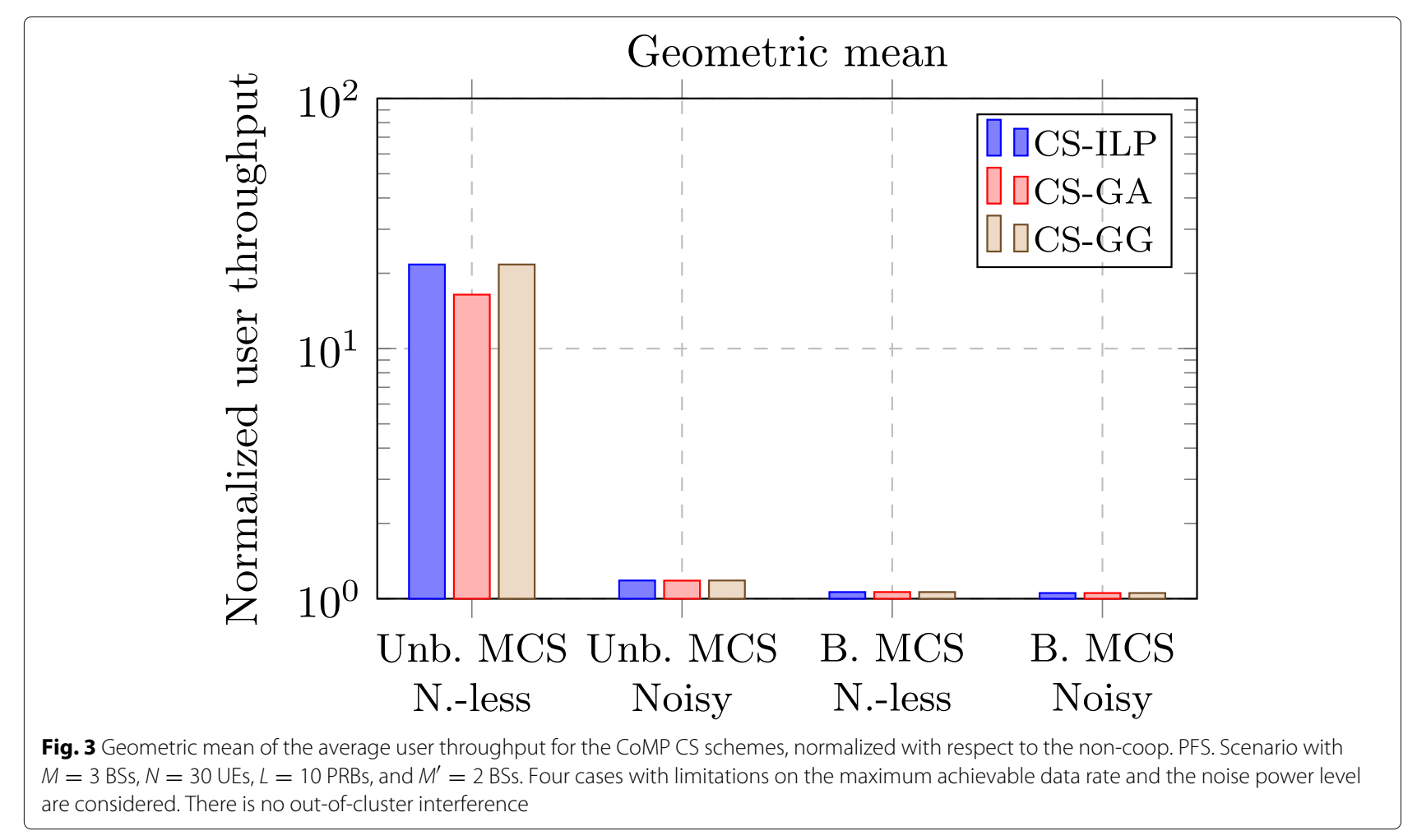

i.e., CS-ILP, CS-GA and CS-GG, are normalized by the resulting user throughput when no cooperative scheduler is applied. Four cases are considered for different combinations of maximum achievable data rate and noise power level, as specified in the horizontal axis. No additional BSs are considered in the network, hence, there is no outof-cluster interference, such that $I_{n, l}^{\mathrm{oc}}=0$. It is observed that under no achievable data rate limitations and noise free receivers, i.e., Unb. MCS and N.-less, significant user throughput gains for both, the cell-edge and the geometric mean, are achieved by the cooperative schemes with respect to the non-coop. PFS. Moreover, the optimality of the proposed CS-ILP formulation is notable, with the CS-GA being unable to obtain the optimal solution as explained in Section 4.3. Due to the unboundedness of the MCS and the noise free decoder assumptions in this case, simultaneously muting the two interfering BSs can significantly increase the UE's data rate. Nevertheless, only muting one interfering BS does not yield sufficient PF metric gain, causing the CS-GA scheme to stop prematurely. Such a limitation of the CS-GA is not present in the proposed CS-GG, which achieves the same optimal performance as the CS-ILP scheme. Once limitations are assumed in either the maximum achievable data rate and/or the noise power level, the observed gains from the CS with muting schemes with respect to the non-coop. PFS approach, vanish. Due to the low number of BSs in the cooperation cluster and given the above mentioned limitations, few users benefit from the simultaneous muting of the two interfering BSs.

The average percentage of muted PRBs per BS, for the four different scheduling schemes and four combinations of maximum achievable data rate and noise power level, is presented in Table 3. The non-coop. PFS does not apply muting, therefore the table contains zero entries for all cases. For the CS with muting schemes, according to Figs. 2 and 3, the average percentage of muted resources per BS reduces when the gain of muting is restricted. It is worth noting that even when the maximum achievable data rate is assumed to be unbounded above and noiseless receivers are considered, the CS-ILP scheme mutes $2 / 3$ of the resources per BS, which means that each BS orthogonally schedules its UEs over $1 / M$-th of the available resources. Further muting resources per $\mathrm{BS}$, reduces the network performance because the user throughput distribution lacks fairness among the BSs. The

Table 3 Average percentage of muted resources per BS

\begin{tabular}{lllll}
\hline $\begin{array}{l}\text { Scheduling } \\
\text { scheme }\end{array}$ & Non-coop. & CS & CS & CS \\
\hline Unb. MCS \& N.-less & 0 & ILP & GA & GG \\
Unb. MCS \& Noisy & 0 & 0.67 & 0.53 & 0.67 \\
B. MCS \& N.-less & 0 & 0.22 & 0.21 & 0.22 \\
B. MCS \& Noisy & 0 & 0.08 & 0.08 & 0.08 \\
\hline
\end{tabular}


value $1 / M$, represents a fundamental limit of the cooperation and agrees with analytical studies presented by Lozano et al. in [26]. Although the performance of the heuristic CS with muting schemes is near-optimal under current practical network conditions, it is envisioned that the evolution of mobile communications introduces for future networks receivers with enhanced capabilities to suppress noise and to support the usage of higher MCSs. Hence, the results in Figs. 2 and 3, provide a reference to the potential gains of these heuristic schemes with respect to the optimal performance obtained with the proposed CS-ILP.

In the following, the more practical scenario with a maximum achievable data rate of $5.4 \mathrm{bits} / \mathrm{symbol}$ and a noise figure of $9 \mathrm{~dB}$, is considered in order to study the performance of the CS with muting schemes, with respect to the cooperation cluster size. For that purpose, a network of seven BSs is simulated, where a single cooperation cluster of variable size, with $M \in\{3, \ldots, 7\}$, is assumed. The BSs outside of the cooperation cluster are assumed to transmit data with maximum transmit power over the complete simulation time, such that $I_{n, l}^{\text {oc }} \geq 0$. Additionally, two alternatives for the number of strongest interfering BSs per UE are considered with $M^{\prime}=M-1$ and $M^{\prime}=2$. Each BS serves 10 UEs over $L=10$ PRBs. The cell-edge throughput, as a function of the cooperation cluster size $M$, is shown in Fig. 4 for the UEs served by the BSs within the cooperation cluster. The presented results are normalized with respect to the user throughput achieved by the same UEs, if the non-coop. PFS is used. In accordance to the previous results, the CS with muting schemes provide gains with respect to a non-cooperative PF scheduler, with an increase in the gains for a larger cooperation cluster size. The reason for such an improvement is the opportunity of further reducing the interference, and thus enhancing the SINR, by increasing the amount of BSs involved in the coordinated scheduling procedures. It is also observable that a larger number of $M^{\prime}$ strongest interfering BSs per UE improves the gains of the CS with muting schemes, at the cost of additional computational complexity and signaling overhead. In agreement with the results presented in Figs. 2 and 3, the greedy algorithm of [25] shows a near-optimal performance under practical conditions, with the proposed CS-GG algorithm performing better than the CS-GA scheme when all possible strongest interfering BSs are considered. Similar results were observed for the geometric mean of the user throughput.

\subsection{CS with muting-potential gains}

In this section, system level simulation results are presented in order to demonstrate the achievable gains of the CS with muting schemes for LTE-Advanced macro-only and heterogeneous networks in an urban deployment. In both cases, $N=630$ UEs are served over $L=10$ PRBs, by $M=21 \mathrm{BSs}$ in the macro-only network and $M=42 \mathrm{BSs}$ in the heterogeneous case where, one pico cell is located within the coverage area of a macro BS with a separation distance of $125 \mathrm{~m}$ from the macro BS. The UEs are



Fig. 4 Average cell-edge user throughput, normalized with respect to the non-coop. PFS, for different cooperation cluster sizes ( $M$ ). Scenario with $N=10 M U E s, L=10 \mathrm{PRBs}$ and $M^{\prime}=\{2, M-1\}$ BSs. There is out-of-cluster interference 
uniformly distributed in the macro-only case, while in the heterogeneous network the UEs are located in a hotspot fashion, where $2 / 3$ of the UEs are deployed in the vicinity of the pico BSs. In the heterogeneous networks cell range expansion is used with a SINR off-set of $6 \mathrm{~dB}$ for the small cells. The out-of-cluster interference is modeled using the wrap-around technique [41], where additional BSs are deployed surrounding the $M$ BSs of interest. Additionally, $\mathrm{CSI}_{\text {lte }}^{\mathrm{R}-11}$ reporting with periodicity of $5 \mathrm{~ms}$ is applied. Full buffer conditions, ideal link adaptation and rank one transmissions are assumed, i.e., all users are always active and demand as much data as possible, there are no decoding errors and only transmit beamforming is applied, respectively. For more information on 3GPPcompliant system level simulations, including channel and path-loss models, the interested reader is referred to [29] (See 3GPP Case 1 and Case 6.2 from Section A.2.1).

The cell-edge and the geometric mean of the user throughput, normalized with respect to the non-coop, PFS, are presented in Fig. 5 for a macro-only, and in Fig. 6 for a heterogeneous network. In terms of the geometric mean, gains are limited to values around $11 \%$ for both cases, macro-only and heterogeneous networks. Additionally, the difference between the proposed schemes, i.e., CS-ILP and CS-GG, and the state-of-the-art CS-GA is negligible. For the cell-edge users, even with the limitation in the number of strongest interfering BSs, the CS with muting schemes achieve a considerable gain in performance, with gains above $40 \%$ being observable. In the case of heterogeneous networks, the cell-edge gain is even higher, due to the presence of a clear strongest interfering BS for the pico UEs, i.e., the macro BS, which is considered to cooperate within the restriction of $M^{\prime}=2$. The proposed generalized greedy algorithm CS-GG, performs better than the scheme in [25], i.e., CS-GA, which follows from the flexibility to muting additional BSs. The average percentages of muted PRBs for the CS with muting schemes in the macro-only and heterogeneous networks are presented in Table 4. One implication of the muted PRBs is the opportunity to save transmit power at the BSs, with the proposed CS-ILP and CS-GG schemes muting more PRBs than the CS-GA scheme.

It is worth to remark that the performance of equivalent decentralized CoMP CS schemes is expected to be upper bounded by their centralized counterparts, as shown in [25]. Hence, the results provided in this work give a reference of the maximum expected performance for the decentralized schemes. A decentralized CoMP CS scheme is proposed and compared with the results in the paper at hand in [42].

Finally, focusing on the proposed parallelized CS-ILP, it is recognizable that the simplifications proposed in Section 4.2, enable the implementation of such a CS with muting approach even for medium to large-size networks. Hence, instead of solving the CS with muting problem by considering the total of $N=630$ UEs per PRB $l \in \mathcal{L}$, only $\left|\mathcal{N}_{l}^{\prime}\right|=136$ and $\left|\mathcal{N}_{l}^{\prime}\right|=213$ UEs were included in average for the macro-only and the heterogeneous network, respectively. That implies a reduction of 78 and $66 \%$ in the problem size, for each of the cases, respectively.



Fig. 5 Cell-edge and geometric mean of the average user throughput, normalized with respect to the non-coop. PFS, for a scenario with $M=21$ $\mathrm{BSS}, N=630 \mathrm{UES}, \mathrm{L}=10 \mathrm{PRBs}$, and $\mathrm{M}^{\prime}=2 \mathrm{BSs}$, with wrap-around technique. Results from system level simulations of a macro-only network 




Fig. 6 Cell-edge and geometric mean of the average user throughput, normalized with respect to the non-coop. PFS, for a scenario with $M=42$ $B S s, N=630 \mathrm{UES}, L=10 \mathrm{PRBs}$, and $M^{\prime}=2 \mathrm{BS}$, with wrap-around technique. Results from system level simulations of a heterogeneous network

\section{Conclusions}

In this paper, the coordinated scheduling with muting problem in the framework of LTE-Advanced networks with a centralized controller has been studied. A novel integer non-linear program formulation has been proposed to solve the problem optimally, where a computationally efficient equivalent integer linear program reformulation has been proposed to extend the applicability of the derived scheme even to large-size networks.

Extensive system level simulation results show that coordinated scheduling with muting can potentially improve the cell-edge user performance, with higher gains in heterogeneous networks. Nevertheless, these gains are limited by the remaining uncoordinated interference and the finite time/frequency/space resources to be shared in the network.

The evaluation of the proposed integer linear program formulation, as well as the state-of-the-art heuristic greedy algorithm, for alternative traffic models in the nonfull buffer case, are recommended for future studies. In the case of low demand, the possibility of reducing residual interference and increasing the degrees of freedom for the cooperation can further enhance the performance gains of the mentioned coordinated scheduling schemes.

Table 4 Average percentage of muted resources

\begin{tabular}{llll}
\hline Network & CS-ILP & CS-GA & CS-GG \\
\hline Macro-only & 0.11 & 0.10 & 0.10 \\
Heterogeneous & 0.13 & 0.08 & 0.09 \\
\hline
\end{tabular}

\section{Acknowledgments}

We acknowledge support by the German Research Foundation and the Open Access Publishing Fund of Technische Universität Darmstadt.

\section{Competing interests}

The authors declare that they have no competing interests.

\section{Publisher's Note}

Springer Nature remains neutral with regard to jurisdictional claims in published maps and institutional affiliations.

\section{Author details}

${ }^{1}$ Communication Systems Group, Darmstadt University of Technology, 64283 Darmstadt, Germany. ${ }^{2}$ Technology Innovation Division, Deutsche Telekom AG 64295 Darmstadt, Germany.

Received: 13 February 2017 Accepted: 22 June 2017

Published online: 11 July 2017

\section{References}

1. SG Kiani, GE Oien, D Gesbert, in 2007 IEEE Wireless Communications and Networking Conference. Maximizing Multicell Capacity Using Distributed Power Allocation and Scheduling, (2007), pp. 1690-1694. doi:10.1109/WCNC.2007.318

2. SG Kiani, D Gesbert, Optimal and distributed scheduling for multicell capacity maximization. IEEE Trans. Wirel. Commun. 7(1), 288-297 (2008)

3. G Boudreau, J Panicker, N Guo, R Chang, N Wang, S Vrzic, Interference coordination and cancellation for $4 \mathrm{G}$ networks. IEEE Commun. Mag. 47(4), 74-81 (2009)

4. Y Yang, Q Zhang, P Shang, J Liu, in 2014 IEEE 25th Annual International Symposium on Personal, Indoor, and Mobile Radio Communication (PIMRC). Interference alignment based coordinated scheduling for uplink small cell enhancement, (2014), pp. 1109-1114. doi:10.1109/PIMRC.2014.7136333

5. Cisco, Cisco Visual Networking Index: Global mobile data traffic forecast update, 2015-2020 (2016). http://www.cisco.com

6. L Zheng, DNC Tse, Diversity and multiplexing: a fundamental tradeoff in multiple-antenna channels. IEEE Trans. Inf. Theory. 49(5), 1073-1096 (2003) 
7. A Paulraj, R Nabar, D Gore, Introduction to space-time wireless communications. (Cambridge University Press, Cambridge, 2003)

8. AB Gershman, ND Sidiropoulos, Space-time processing for MIMO communications (John Wiley \& Sons, Chichester, 2005)

9. J Lee, J-K Han, J Zhang, MIMO Technologies in 3GPP LTE and LTE-Advanced. EURASIP J. Wirel. Commun. Netw. 2009, 302092 (2009)

10. JG Andrews, Seven ways that HetNets are a cellular paradigm shift. IEEE Commun. Mag. 51(3), 136-144 (2013)

11. YL Lee, TC Chuah, J Loo, A Vinel, Recent advances in radio resource management for heterogeneous LTE/LTE-A networks. IEEE Commun. Surv. Tutorials. 16(4), 2142-2180 (2014)

12. D Lopez-Perez, I Güvenc, G de la Roche, M Kounttouris, TQS Quek, J Zhang, Enhanced intercell interference coordination challenges in heterogeneous networks. IEEE Wirel. Commun. 18(3), 22-30 (2011)

13. R Irmer, H Droste, P Marsch, M Grieger, G Fettweis, S Brueck, H-P Mayer, L Thiele, V Jungnickel, Coordinated multipoint: concepts, performance, and field trial results. IEEE Commun. Mag. 49(2), 102-111 (2011)

14. J Lee, Y Kim, H Lee, BL Ng, D Mazzarese, J Liu, W Xiao, Y Zhou, Coordinated multipoint transmission and reception in LTE-Advanced systems. IEEE Commun. Mag. 50(11), 44-50 (2012)

15. EG Larsson, EA Jorswieck, Competition versus cooperation on the MISO interference channel. IEEE J Sel. Areas Commun. 26(7), 1059-1069 (2008)

16. P Baracca, F Boccardi, N Benvenuto, A dynamic clustering algorithm for downlink CoMP systems with multiple antenna UEs. EURASIP J. Wirel. Commun. Netw. 2014, 125 (2014)

17. A Beylerian, T Ohtsuki, Multi-point fairness in resource allocation for C-RAN downlink COMP transmission. EURASIP J. Wirel.Commun. Netw. 2016, 12 (2016)

18. OD Ramos-Cantor, J Belschner, M Pesavento, in 2016 IEEE 17th International Workshop on Signal Processing Advances in Wireless Communications (SPAWC). Improved link adaptation with coordinated scheduling in non-fully loaded wireless networks, (2016), pp. 1-6. doi:10.1109/SPAWC.2016.7536902

19. L Su, C Yang, S Han, The value of channel prediction in CoMP systems with large Backhaul latency. IEEE Trans. Commun. 61(11), 4577-4590 (2013)

20. H-L Määttänen, K Hämäläinen, J Venäläinen, K Schober, M Enescu, M Valkama, System-level performance of LTE-Advanced with joint transmission and dynamic point selection schemes. EURASIP J. Adv. Signal Process. 2012, 247 (2012)

21. B Mondal, E Visotsky, TA Thomas, X Wang, A Ghosh, in 2012 IEEE 23rd International Symposium on Personal, Indoor and Mobile Radio Communications - (PIMRC). Performance of downlink comp in LTE under practical constraints, (2012), pp. 2049-2054. doi:10.1109/PIMRC.2012.6362691

22. A Barbieri, P Gaal, S Geirhofer, T Ji, D Malladi, Y Wei, F Xue, in 2012 Information Theory and Applications Workshop. Coordinated downlink multi-point communications in heterogeneous cellular networks, (2012), pp. 7-16. doi:10.1109/ITA.2012.6181826

23. A Davydov, G Morozov, I Bolotin, A Papathanassiou, in 2013 IEEE Globecom Workshops (GC Wkshps). Evaluation of Joint Transmission CoMP in C-RAN based LTE-A HetNets with large coordination areas, (2013), pp. 801-806. doi:10.1109/GLOCOMW.2013.6825087

24. R Agrawal, A Bedekar, S Kalyanasundaram, N Arulselvan, T Kolding, H Kroener, in 2014 IEEE 79th Vehicular Technology Conference (VTC Spring). Centralized and Decentralized Coordinated Scheduling with Muting, (2014), pp. 1-5. doi:10.1109/NTCSpring.2014.7022856

25. S Gulati, S Kalyanasundaram, P Nashine, B Natarajan, R Agrawal, A Bedekar, in 2015 IEEE 82nd Vehicular Technology Conference (VTC2015-Fall). Performance Analysis of Distributed Multi-Cell Coordinated Scheduler, (2015), pp. 1-5. doi:10.1109/NTCFall.2015.7391069

26. A Lozano, RW Heath Jr, JG Andrews, Fundamental limits of cooperation. IEEE Trans. Inf. Theory. 59(9), 5213-5226 (2013)

27. C Galiotto, N Marchetti, L Doyle, The role of the total transmit power on the linear area spectral efficiency gain of cell-splitting. IEEE Commun. Lett. 17(12), 2256-2259 (2013)

28. Q Ye, B Rong, Y Chen, M Al-Shalash, C Caramanis, JG Andrews, User association for load balancing in heterogeneous cellular networks. IEEE Trans. Wirel. Commun. 12(6), 2706-2716 (2013)

29. 3rd Generation Partnership Project, TR 36.814 v9.0.0 — Further advancements for E-UTRA physical layer aspects (Release 9) (2010)
30. E Dahlman, S Parkvall, J Sköld, 4G: LTE/LTE-Advanced for mobile broadband, 2nd edn. (Elsevier, Oxford, 2014)

31. 3rd Generation Partnership Project, TS 36.213 v11.3.0_physical layer procedures (Release 11) (2013)

32. HJ Zhu, RHM Hafez, Scheduling schemes for multimedia service in wireless OFDM systems. IEEE Wirel. Commun. 14(5), 99-105 (2007)

33. J Wu, NB Mehta, AF Molisch, J Zhang, Unified Spectral Efficiency analysis of cellular systems with channel-aware schedulers. IEEE Trans. Commun. 59(12), 3463-3474 (2011)

34. E Balas, Projection, Lifting and extended formulation in integer and combinatorial optimization. Springer Ann. Oper. Res. 140(1), 125-161 (2005)

35. 3rd Generation Partnership Project, TR 25.996 v11.0.0—Spatial channel model for Multiple Input Multiple Output (MIMO) simulations (Release 11) (2012)

36. 3rd Generation Partnership Project, TR 36.872 v12.1.0 — Small cell enhancements for E-UTRA and E-UTRAN - Physical layer aspects (Release 12) (2013)

37. 3rd Generation Partnership Project, TR 36.874 v12.0.0-Coordinated multi-point operation for LTE with non-ideal backhaul (Release 12) (2013)

38. Motorola, R1-060877 - Frequency Domain Scheduling for E-UTRA (2006). http://www.3gpp.org

39. S Sesia, M Baker, I Toufik, LTE-the UMTS Long Term Evolution: from theory to practice, 2nd edn. (John Wiley \& Sons, Chichester, 2011)

40. Y Cheng, A Philipp, M Pesavento, in 2012 Proceedings of the 20th European Signal Processing Conference (EUSIPCO). Dynamic rate adaptation and multiuser downlink beamforming using mixed integer conic programming, (2012), pp. 824-828

41. Y Yoon, L Duan, F Khaleghi, ACK Soong, Basic operation of the wrap-around technique for system-level simulation. 3GPP2 Technical Report (2002)

42. OD Ramos-Cantor, M Pesavento, in 2017 IEEE 18th International Workshop on Signal Processing Advances in Wireless Communications (SPAWC). Decentralized coordinated scheduling with muting in LTE-Advanced networks, (2017), pp. 1-5

\section{Submit your manuscript to a SpringerOpen ${ }^{\circ}$ journal and benefit from:}

- Convenient online submission

Rigorous peer review

- Open access: articles freely available online

- High visibility within the field

- Retaining the copyright to your article

Submit your next manuscript at $>$ springeropen.com 